\title{
Coupling Coefficients for the Octahedral Group with Orthorhombic Components
}

\author{
LAWRENCE L. LOHR, JR. \\ Department of Chemistry, University of Michigan, Ann Arbor, Michigan 48104
}

Received October 6, 1969

\begin{abstract}
A tabulation is given of the vector coupling coefficients for the octahedral group, where the components of the degenerate representations have been defined with respect to a two-fold octahedral axis. Coefficients are given for both real and complex choices of the components, and should be useful in describing nearly octahedral transition metal complexes with orthorhombic perturbations.

Tabellen von Vektor-Kopplungskoeffizienten für die oktaedrische Gruppe werden angegeben, wobei die Komponenten der entarteten Darstellungen bezüglich der zweizähligen Achsen im Oktaeder definiert sind. Die Koeffizienten werden sowohl für die Wahl von reellen als auch komplexen Basisfunktionen angeführt und sollten zur Beschreibung von fast-oktaedrischen Übergangsmetall-Komplexen mit orthorhombischen Störungen von Vorteil sein.

Table des coefficients de couplage vectoriel pour le groupe octaédrique, les composantes des représentations dégénérées étant définies par rapport à un axe octaédrique binaire. Les coefficients sont donnés pour un choix de composantes réelles ou complexes; ils devraient être utiles pour la description des complexes de métaux de transition presque octaédriques avec des perturbations orthorhombiques.
\end{abstract}

\section{Introduction}

The quantum mechanical description of transition metal complexes is, as with atoms, greatly facilitated by the use of reduced matrix elements and vector coupling coefficients. This group theoretical algebra has been developed for the octahedral group by Tanabe and Sugano [1], Tanabe and Kamimura [2], and Griffith [3], their work paralleling in many ways that for atoms described by Fano and Racah [4]. The basic relation is the Wigner-Eckart theorem, which in Griffith's notation [3] takes the form

$$
\left\langle a \alpha\left|g_{\beta}^{b}\right| a^{\prime} \alpha^{\prime}\right\rangle=\left\langle a\left\|g^{b}\right\| a^{\prime}\right\rangle V\left(\begin{array}{lll}
a & a^{\prime} & b \\
\alpha & \alpha^{\prime} & \beta
\end{array}\right),
$$

where the matrix element of an operator $g$ transforming as component $\beta$ of representation $b$ connects states transforming as components $\alpha$ and $\alpha^{\prime}$ of the representations $a$ and $a^{\prime}$ respectively. The double-bar reduced matrix element is independent of the component labels $\alpha, \alpha^{\prime}$, and $\beta$, while the $V$ coefficient, analogous to a $3-j$ symbol, is completely determined by group-theoretic considerations. The relation (1) holds for real components, with some modification if complex components are chosen ${ }^{1}$.

Griffith tabulates [3] four sets of $V$ coefficients for use in describing systems with octahedral (or tetrahedral) symmetry. These differ only in the choice of

${ }^{1}$ Ref. [3], p. 21; the change consists of replacing $\alpha$ in $V$ by $-\alpha$ and multiplying the expression by a phase factor $[-1]^{a+\alpha}$, where $[-1]^{\xi}=1$ if $\xi$ is $A_{1}, A_{2}, E$, or a component of $E,-1$ if $\xi$ is $T_{1}$ or $T_{2}$, and $(-1)^{\xi}$ if $\xi$ is a component of $T_{1}$ or $T_{2}$. 
components for the degenerate representations. These choices are real tetragonal, real trigonal, complex tetragonal, and complex trigonal. The tetragonal and trigonal systems are based on the choice of a four-fold or a three-fold quantization axis, respectively. Complex components are useful, as with atoms, in describing systems in magnetic fields.

We have on several occasions found it useful to have available $V$ coefficients similar to the above types, but defined instead with respect to a two-fold axis of an octahedron. One example is in the description of cis- $\mathrm{MA}_{4} \mathrm{~B}_{2}$ complexes, such as cis- $\mathrm{Mn}\left(\mathrm{OH}_{2}\right)_{4} \mathrm{Cl}_{2}$, present in crystalline $\mathrm{MnCl}_{2} \cdot 4 \mathrm{H}_{2} \mathrm{O}$. While the molecular symmetry is essentially $C_{2 v}$ (less in the solid), it is still convenient to use an octahedral description with low-symmetry fields defined relative to an octahedral two-fold axis passing through the $\mathrm{Cl}-\mathrm{Cl}$ edge. Another example is the antiferromagnet $\mathrm{MnF}_{2}$, having the rutile crystal structure with $D_{2 h}$ cation site symmetry. Here the antiferromagnetic exchange field of over $500 \mathrm{KGauss}$ is parallel to the $c$ axis of the tetragonal crystal and hence passes along a $C_{2}$ axis of the nearly octahedral $\mathrm{MnF}_{6}^{-4}$ unit, bisecting a $\mathrm{F}-\mathrm{F}$ edge. In a recent calculation [5] of magnetic dipole oscillator strengths for the crystal-field transitions in $\mathrm{MnF}_{2}$, we used the $V$ coefficients presented here to check the results obtained separately by a transformation of $f$-values computed in a tetragonal component system.

\section{Procedure}

In order to calculate $V$ coefficients with orthorhombic components we must define the new components in terms of a unitary transformation of components for which the $V$ are known, say the real tetragonal case. The new $V$ are obtained from the old $V$ by Griffith's transformation ${ }^{2}$.

$$
V\left(\begin{array}{ccc}
a & b & c \\
\alpha^{\prime} & \beta^{\prime} & \gamma^{\prime}
\end{array}\right)=\sum_{\alpha \beta \gamma} A_{\alpha^{\prime} \alpha} B_{\beta^{\prime} \beta} C_{\gamma^{\prime} \gamma} V\left(\begin{array}{lll}
a & b & c \\
\alpha & \beta & \gamma
\end{array}\right),
$$

where a function (state or operator) transforming as the $\alpha^{\prime}$ component of irreducible representation $\boldsymbol{a}$ is given by

$$
f_{\alpha^{\prime}}^{a}=\sum_{\alpha} A_{\alpha^{\prime} \alpha} f_{\alpha}^{a}
$$

with similar expressions for $f_{\beta^{\prime}}^{b}$ and $f_{\gamma^{\prime}}^{c}$.

If $x, y$, and $z$ denote real tetragonal components of a $T_{1}$ or $T_{2}$ representation, then real orthorhombic components $x^{\prime}, y^{\prime}$, and $z^{\prime}$ are given by the transformation

$$
\left(\begin{array}{l}
x^{\prime} \\
y^{\prime} \\
z^{\prime}
\end{array}\right)=\left(\begin{array}{lll}
2^{-1 / 2} & 2^{-1 / 2} & 0 \\
0 & 0 & 1 \\
2^{-1 / 2} & -2^{-1 / 2} & 0
\end{array}\right)\left(\begin{array}{l}
x \\
y \\
z
\end{array}\right)
$$

with the complex orthorhombic components 1,0 , and -1 then given by

$$
\left(\begin{array}{r}
1 \\
0 \\
-1
\end{array}\right)=\left(\begin{array}{ccc}
-i / 2^{1 / 2} & 2^{-1 / 2} & 0 \\
0 & 0 & i \\
i / 2^{1 / 2} & 2^{-1 / 2} & 0
\end{array}\right)\left(\begin{array}{l}
x^{\prime} \\
y^{\prime} \\
z^{\prime}
\end{array}\right)
$$

\footnotetext{
${ }^{2}$ Ref. [3], p. 18; if the components $\alpha^{\prime}, \beta^{\prime}$, and $\gamma^{\prime}$ are complex, replace $A_{\alpha^{\prime} \alpha}$, in (2) by its complex conjugate, which from (5) is simply $A_{-\alpha^{\prime} \alpha}$.
} 
The elements of the square arrays in (4) or (5) comprise the $A_{\alpha^{\prime} \alpha}, B_{\beta^{\prime} \beta}$, or $C_{\gamma^{\prime} y}$ of (2). No transformations were made for the representations $A_{1}, A_{2}$, and $E$. This means that the two components of $E$ are taken as $\theta, \varepsilon$ functions, corresponding to $3 z^{2}-r^{2}$ and $x^{2}-y^{2}$, respectively. This can be done because the particular two-fold axis chosen for $z^{\prime}$ lies in the $x y$ plane, and is the axis $(x-y)$. In the new coordinate system $x^{\prime}$ is also a two-fold axis, but $y^{\prime}$ is a four-fold axis. Thus $\theta$ and $\varepsilon$ are irreducible representations of the sub-group $C_{2 v}$ with $z^{\prime}$ as the two-fold axis.

\section{Results}

Coupling coefficients involving $T_{1}$ and $T_{2}$ representations with real orthorhombic components are presented in Table 1 . The component labels $x, y$, and $z$ in Table 1 refer to $x^{\prime}, y^{\prime}$, and $z^{\prime}$ of Eq. (4). Since no transformation was made on the components of $A_{1}, A_{2}$, or $E$ representations, $V$ coefficients involving only these representations, namely $V$ 's of types $A_{1}^{3}, A_{1} A_{2}^{2}, A_{1} E^{2}, A_{2} E^{2}$, and $E^{3}$, are unchanged from their real tetragonal values [3]. For completeness these are: $V\left(A_{1}^{3}\right)=V\left(A_{1} A_{2}^{2}\right)=1 ; V\left(A_{1} E^{2}\right)=2^{-1 / 2} \delta_{\beta \gamma}$, where $\beta, \gamma=\theta$ or $\varepsilon ; V\left(A_{2} E^{2}\right)= \pm 2^{-1 / 2}$ for $\beta \gamma=\theta \varepsilon$ and $\varepsilon \theta$ respectively; and $V\left(E^{3}\right)=1 / 2$ for $\alpha \beta \gamma=\theta \varepsilon \varepsilon, \varepsilon \theta \varepsilon$, or $\varepsilon \varepsilon \theta$, but $-1 / 2$ for $\alpha \beta \gamma=\theta \theta \theta$. All real and complex orthorhombic V's satisfy Griffith's

Table 1. Real orthorhombic component system ${ }^{\mathrm{a}, \mathrm{b}}$

\begin{tabular}{|c|c|c|c|}
\hline$T_{2}$ & $T_{2}$ & $T_{2}$ & \\
\hline$T_{1}$ & $T_{1}$ & $T_{2}$ & $V$ \\
\hline$x$ & $x$ & $y$ & $-6^{-1 / 2}$ \\
\hline$z$ & $z$ & $y$ & $6^{-1 / 2}$ \\
\hline$x$ & $y$ & $x$ & $-6^{-1 / 2}$ \\
\hline$z$ & $y$ & $z$ & $6^{-1 / 2}$ \\
\hline$y$ & $x$ & $x$ & $-6^{-1 / 2}$ \\
\hline$y$ & $z$ & $z$ & $6^{-1 / 2}$ \\
\hline$E$ & $T_{2}$ & $T_{2}$ & 0 \\
\hline$E$ & $T_{1}$ & $T_{1}$ & $V$ \\
\hline$\theta$ & $x$ & $x$ & $12^{-1 / 2}$ \\
\hline$\theta$ & $z$ & $z$ & $12^{-1 / 2}$ \\
\hline$\theta$ & $y$ & $y$ & $-3^{-1 / 2}$ \\
\hline$\varepsilon$ & $x$ & $z$ & $-1 / 2$ \\
\hline$\varepsilon$ & $z$ & $x$ & $-1 / 2$ \\
\hline$E$ & $T_{1}$ & $T_{2}$ & $V$ \\
\hline$\varepsilon$ & $y$ & $y$ & $3^{-1 / 2}$ \\
\hline$\varepsilon$ & $x$ & $x$ & $-12^{-1 / 2}$ \\
\hline$\varepsilon$ & $z$ & $z$ & $-12^{-1 / 2}$ \\
\hline$\theta$ & $x$ & $z$ & $-1 / 2$ \\
\hline$\theta$ & $z$ & $x$ & $-1 / 2$ \\
\hline
\end{tabular}


Table 2. Complex orthorhombic component system ${ }^{\mathrm{a}}$

\begin{tabular}{|c|c|c|c|c|c|c|c|}
\hline$T_{2}$ & $T_{2}$ & $T_{2}$ & & $E$ & $T_{2}$ & $T_{2}$ & \\
\hline$T_{1}$ & $T_{1}$ & $T_{2}$ & $V$ & $E$ & $T_{1}$ & $T_{1}^{t}$ & $V$ \\
\hline 0 & 0 & \pm 1 & $-12^{-1 / 2}$ & $\theta$ & 0 & 0 & $-12^{-1 / 2}$ \\
\hline 0 & \pm 1 & 0 & $-12^{-1 / 2}$ & $\theta$ & 1 & 1 & $-3^{1 / 2} / 4$ \\
\hline \pm 1 & 0 & 0 & $-12^{-1 / 2}$ & $\theta$ & -1 & -1 & $-3^{1 / 2} / 4$ \\
\hline 1 & 1 & 1 & $3^{1 / 2} / 4$ & $\theta$ & 1 & -1 & $-48^{-1 / 2}$ \\
\hline 1 & 1 & -1 & $-48^{-1 / 2}$ & $\theta$ & -1 & 1 & $-48^{-1 / 2}$ \\
\hline 1 & -1 & 1 & $-48^{-1 / 2}$ & $\varepsilon$ & \pm 1 & 0 & $\mp 8^{-1 / 2}$ \\
\hline 1 & -1 & -1 & $-48^{-1 / 2}$ & $\varepsilon$ & 0 & \pm 1 & $\mp 8^{-1 / 2}$ \\
\hline-1 & 1 & 1 & $-48^{-1 / 2}$ & & & & \\
\hline-1 & 1 & -1 & $-48^{-1 / 2}$ & $E$ & $T_{1}$ & $T_{2}$ & $V$ \\
\hline-1 & -1 & 1 & $-48^{-1 / 2}$ & & & & \\
\hline \multirow[t]{2}{*}{-1} & -1 & -1 & $3^{1 / 2 / 4}$ & $\varepsilon$ & 0 & 0 & $12^{-1 / 2}$ \\
\hline & & & & $\varepsilon$ & 1 & 1 & $3^{1 / 2} / 4$ \\
\hline$A_{1}$ & $T_{1}$ & $T_{1}$ & $V^{b}$ & $\varepsilon$ & -1 & -1 & $3^{1 / 2} / 4$ \\
\hline$a_{1}$ & 1 & -1 & $3-1 / 2$ & $\varepsilon$ & 1 & -1 & $\begin{array}{l}48^{-1 / 2} \\
8^{-1 / 2}\end{array}$ \\
\hline$a_{1}$ & 0 & 0 & $-3^{-1 / 2}$ & $\theta$ & $\begin{array}{l}-1 \\
+1\end{array}$ & $\begin{array}{l}1 \\
0\end{array}$ & $\begin{array}{r}48-1 / 2 \\
\mp \quad 8^{-1 / 2}\end{array}$ \\
\hline$a_{1}$ & -1 & 1 & $3^{-1 / 2}$ & $\theta$ & 0 & \pm 1 & $\mp 8^{-1 / 2}$ \\
\hline
\end{tabular}

symmetry with respect to permutation of columns; permutation introduces the phase factor $(-1)$ raised to a power $(a+b+c)$, where $(-1)$ to the $A_{1}, E$, or $T_{2}$ is defined to be +1 , while $(-1)$ to the $A_{2}$ or $T_{1}$ is defined to be -1 .

Similarly, coupling coefficients involving $T_{1}$ or $T_{2}$ representations with complex orthorhombic components are given in Tables 2 and 3 . Coefficients involving only $A_{1}, A_{2}$, and $E$ representations are unchanged from their complex tetragonal values, and are equal to their real tetragonal values. As in the complex tetragonal (but not complex trigonal) case, all $V$ for complex orthorhombic components are real.

Several of the $V$ coefficients are expressed in terms of the Levi-Civita tensor $\varepsilon$, whose elements $\varepsilon_{\alpha \beta \gamma}$ are 0 if any two $\alpha \beta \gamma$ are equal, 1 if $\alpha \beta \gamma$ is an even permutation of $x y z$ (or of $1,0,-1$ ), and -1 if an odd permutation.

\section{References}

1. Tanabe, Y., Sugano, S.: J. physic. Soc. Japan 9, 753 (1954).

2. - Kamimura, H.: J. physic. Soc. Japan 13, 394 (1958).

3. Griffith, J. S.: The irreducible tensor method for molecular symmetry groups. Englewood Cliffs: Prentice-Hall, Inc., N. J. 1962.

4. Fano, U., Racah, G.: Irreducible tensorial sets. New York: Academic Press 1959.

5. Meltzer, R. S., Lohr, L. L., Jr.: J. chem. Physics 49, 541 (1968).

Professor Lawrence L. Lohr, Jr.

Department of Chemistry

The University of Michigan

Ann Arbor, Michigan 48104, USA 